\title{
Certain Class of Analytic Functions with respect to Symmetric Points Defined by Q-Calculus
}

\author{
K. R. Karthikeyan $\mathbb{D}^{1},{ }^{1}$ G. Murugusundaramoorthy $\mathbb{D}^{2},{ }^{2}$ S. D. Purohit $\mathbb{D}^{3},{ }^{3}$ and D. L. Suthar $\mathbb{I D}^{4}$ \\ ${ }^{1}$ Department of Applied Mathematics and Science, \\ National University of Science \& Technology (By Merger of Caledonian College of Engineering and Oman Medical College), \\ Muscut, Oman \\ ${ }^{2}$ Department of Mathematics, School of Advanced Sciences, Vellore Institute of Technology Deemed to be University, Vellore, \\ Tamilnadu, India \\ ${ }^{3}$ Department of HEAS (Mathematics), Rajasthan Technical University, Kota, India \\ ${ }^{4}$ Department of Mathematics, Wollo University, P. O. Box: 1145, Amhara Region, Ethiopia
}

Correspondence should be addressed to D. L. Suthar; dlsuthar@gmail.com

Received 14 June 2021; Accepted 14 August 2021; Published 26 August 2021

Academic Editor: Firdous A. Shah

Copyright (c) 2021 K. R. Karthikeyan et al. This is an open access article distributed under the Creative Commons Attribution License, which permits unrestricted use, distribution, and reproduction in any medium, provided the original work is properly cited.

In this study, we familiarise a novel class of Janowski-type star-like functions of complex order with regard to $(j, k)$-symmetric points based on quantum calculus by subordinating with pedal-shaped regions. We found integral representation theorem and conditions for starlikeness. Furthermore, with regard to $(j, k)$-symmetric points, we successfully obtained the coefficient bounds for functions in the newly specified class. We also quantified few applications as special cases which are new (or known).

\section{Definitions and Preliminaries}

The set of all analytic functions constructed on the unit disc $\mathbf{U}=\{z \in \mathbb{C}:|z|<1\}$ is symbolised by $\mathscr{H}(\mathbf{U})$. Also, $\mathscr{A}$ indicates the subclass of $\mathscr{H}(\mathbf{U})$ that has a Taylor series representation:

$$
f(\xi)=\xi+\sum_{n=2}^{\infty} a_{n} \xi^{n}, \quad(\xi \in \mathbf{U}=\{\xi:|\xi|<1\}) .
$$

The family of functions $f \in \mathscr{A}$ that are univalent in $\mathbf{U}$ is represented by $\delta$. This is well established that if $f(\xi)$, assume by $(1)$, is in $\mathcal{S}$, then $\left[f\left(\xi^{k}\right)\right]^{1 / k}$ ( $k$ is a positive integer) is consequently in $\delta$.

Definition 1 (see [1], Definition 3). Assume $k$ is a positive integer. A domain $\mathbb{D}$ is known to be $k$-fold symmetric if a rotation of $\mathbb{D}$ about the origin through an angle $2 \pi / k$ carries $\mathbb{D}$ onto itself. For $\mathbf{U}$, a function $f$ is said to be $k$-fold symmetric if and only if for each $\xi$ in $\mathbf{U}$

$$
f\left(e^{2 \pi i / k} \xi\right)=e^{2 \pi i / k} f(\xi)
$$

$\mathscr{F}_{k}$ represents the family including all $k$-fold symmetric functions.

The concept of $k$-symmetrical function was protracted to so-called $(j, k)$-symmetrical function by Liczberski and Połubiński in [2]. To be specific, a function $f(\xi)$ is reported for being $(j, k)$-symmetrical if

$$
f(\varepsilon \xi)=\varepsilon^{j} f(\xi), \quad(\xi \in \mathbf{U}),
$$

where $k \geq 2$ is a fixed integer, $j=0,1,2, \ldots, k-1$ and $\varepsilon=\exp (2 \pi i / k)$. The family of $(j, k)$-symmetrical functions will indeed be indicated by $\mathscr{F}_{k}^{j}$. We believe that $\mathscr{F}_{2}^{1}, \mathscr{F}_{2}^{0}$, and $\mathscr{F}_{k}^{1}$ are quite well groups of odd functions, even functions, and $k$-symmetrical functions. Consider the subsequent equivalence demarcate $f_{j, k}(\xi)$ as well

$$
f_{j, k}(\xi)=\frac{1}{k} \sum_{\nu=0}^{k-1} \frac{f\left(\varepsilon^{v} \xi\right)}{\varepsilon^{v j}}, \quad(f \in \mathscr{A} ; k=1,2, \ldots ; j=0,1,2, \ldots,(k-1)) .
$$


It is evident that $f_{j, k}(\xi)$ is a linear operator from $\mathbf{U}$ into $\mathrm{U}$. If $v$ is an integer, then the subsequent assumptions result directly from (4):

$$
\begin{aligned}
& f_{j, k}\left(\varepsilon^{v} \xi\right)=\varepsilon^{\nu j} f_{j, k}(\xi), \\
& f_{j, k}^{\prime}\left(\varepsilon^{v} \xi\right)=\varepsilon^{v j-v} f_{j, k}^{\prime}(\xi)=\frac{1}{k} \sum_{\nu=0}^{k-1} \frac{f^{\prime}\left(\varepsilon^{\nu} \xi\right)}{\varepsilon^{v j-\nu}} \\
& f_{j, k}^{\prime \prime}\left(\varepsilon^{v} \xi\right)=\varepsilon^{v j-2 v} f_{j, k}^{\prime \prime}(\xi)=\frac{1}{k} \sum_{\nu=0}^{k-1} \frac{f^{\prime \prime}\left(\varepsilon^{\nu} \xi\right)}{\varepsilon^{v j-2 v}} .
\end{aligned}
$$

Let the function $f \in \mathscr{A}$ provided by (1) and $g \in \mathscr{A}$ of the form $g(\xi)=\xi+\sum_{n=2}^{\infty} \Upsilon_{n} \xi^{n}$, the Hadamard product (or convolution) of these two functions is indicated by

$$
\mathscr{H}(\xi):=(f * g)(\xi):=\xi+\sum_{n=2}^{\infty} a_{n} \Upsilon_{n} \xi^{n}, \quad \xi \in \mathbf{U} .
$$

Using Hadamard product, various authors studied the univalent function theory in dual with the theory of special functions, see [3-5] and references provided therein. Throughout this whole article, we will assume that $k \in \mathbb{N}$, $\varepsilon=\exp (2 \pi i / k)$, and

$$
\mathscr{H}_{j, k}(\xi)=\frac{1}{k} \sum_{\nu=0}^{k-1} \varepsilon^{-v j}\left[(f * g)\left(\varepsilon^{\nu} \xi\right)\right]=\xi+\cdots,
$$

where

$$
f, g \in \mathscr{A} ; k=1,2, \ldots ; j=0,1,2, \ldots,(k-1) .
$$

From (7), we, thus, have

$$
\begin{aligned}
\mathscr{H}_{j, k}(\xi) & =\sum_{n=1}^{\infty} a_{n} \Upsilon_{n} \Delta_{n, j} \xi^{n}, \quad\left(a_{1}=\Upsilon_{1}=1\right), \\
\Lambda_{n, j} & =\frac{1}{k} \sum_{v=0}^{k-1} \varepsilon^{(n-j) v} .
\end{aligned}
$$

The investigation of $q$-calculus ( $q$ stands for quantum) fascinated and inspired many scholars due its use in various areas of the quantitative sciences. Jackson $[6,7]$ was among the key contributors of all the scientists who introduced and developed the $q$-calculus theory. Just like $q$-calculus was used in other mathematical sciences, the formulations of this idea are commonly used to examine the existence of various structures of function theory. Though it is the first article in which a link was established between certain geometric nature of the analytic function and the $q$-derivative operator and the usage of $q$-calculus in function theory, a solid and comprehensive foundation is given in [8] by Srivastava. After this development, many researchers introduced and studied some useful operators in $q$-analog with the applications of convolution concepts. For example, Kanas and Raducanu [9] established the $q$-differential operator and then examined the behavior of this operator in function theory. For more applications of this operator, see $[10,11]$.
For $f \in \mathscr{A}$ assumed by (1) and $0<q<1$, the Jackson's $q$-derivative operator or $q$-difference operator for $f \in \mathscr{A}$ is specified under (see [12-14])

$$
\mathfrak{D}_{q} f(\xi):= \begin{cases}f^{\prime}(0), & \text { if } \xi=0, \\ \frac{f(\xi)-f(q \xi)}{(1-q) \xi}, & \text { if } \xi \neq 0 .\end{cases}
$$

From (10), if $f$ is assumed as in (1), we can effortlessly see that

$$
\mathfrak{D}_{q} f(\xi)=1+\sum_{n=2}^{\infty}[n]_{q} a_{n} \xi^{n-1}
$$

for $\xi \neq 0$, provided the $q$-integer number $[n]_{q}$ is represented by

$$
[n]_{q}:=\frac{1-q^{n}}{1-q},
$$

and take into consideration $\lim _{q \longrightarrow 1^{-}} \mathfrak{D}_{q} f(\xi)=f^{\prime}(\xi)$. During our study, we let signify

$$
\left([n]_{q}\right)_{\kappa}:=[n]_{q}[n+1]_{q}[n+2]_{q} \ldots[n+\kappa-1]_{q} .
$$

The $q$-Jackson integral is defined by (see [6])

$$
I_{q}[f(\xi)]:=\int_{0}^{\xi} f(t) \mathrm{d}_{q} t=\xi(1-q) \sum_{k=0}^{\infty} q^{k} f\left(\xi q^{k}\right) .
$$

If the $q$-series converges, further witness that

$$
\begin{aligned}
& \mathfrak{D}_{q} I_{q} f(\xi)=f(\xi), \\
& I_{q} \mathfrak{D}_{q} f(\xi)=f(\xi)-f(0),
\end{aligned}
$$

where the second equality grasps if $f$ is continuous at $\xi=0$.

Let the classes of star-like functions of order $\eta(0 \leq \eta<1)$ and convex functions of order $\eta(0 \leq \eta<1)$ are symbolised by $\mathcal{S}^{*}(\eta)$ and $\mathscr{C}(\eta)$, respectively. In $\mathscr{A}$, we categorize the collection $\mathbf{P}$ of functions $p(\xi) \in \mathscr{A}$ with $p(0)=1$ and $\mathfrak{R} p(\xi)>0$. The functions in the $\mathbf{P}$ class are not univalent.

With $\mathbf{U}$, let $f, g$ be analytic. The function $f$ is said to be subordinate to $g$ in $\mathbf{U}$ if the Schwarz function $\omega(\xi)$ exists in $\mathbf{U}$ such that $|\omega(\xi)|<|\xi|$ and $f(\xi)=g(\omega(\xi))$, as shown through $f \prec g$. Whenever $g$ is univalent in $\mathbf{U}$, consequently the subordination is identical to $f(0)=g(0)$ and $f(\mathbf{U}) \subset g(\mathbf{U})$.

Using the concept of subordination for holomorphic functions, Ma and Minda [15] proposed the classes:

$$
\begin{aligned}
& \mathcal{S}^{*}(\psi)=\left\{f \in \mathscr{A}: \frac{\xi f^{\prime}(\xi)}{f(\xi)} \prec \psi\right\}, \\
& \mathscr{C}(\psi)=\left\{f \in \mathscr{A}:\left(1+\frac{\xi f^{\prime \prime}(\xi)}{f^{\prime}(\xi)}\right) \prec \psi\right\},
\end{aligned}
$$

where $\psi \in \mathbf{P}$ with $\psi^{\prime}(0)>0$ maps $\mathbf{U}$ onto a region star-like with respect to 1 and symmetric with respect to the real axis. By making a choice $\psi$ to map unit disc on to some specific regions such as cardioid, parabolas, lemniscate of Bernoulli, and booth lemniscate in the right-half of the complex plane, 
various interesting subclasses of star-like and convex functions could be gained well.

Lots of fascinating subclasses of star-like and convex functions may be constructed by using $\psi$ to map unit disc on to particular areas such as cardioid, parabolas, lemniscate of Bernoulli, and booth lemniscate on the right-half of the complex plane.

For arbitrary fixed numbers $C, D,-1<C \leq 1$, and $-1 \leq$ $D<C$, we express through $\mathbf{P}(C, D)$ the family of functions $p(\xi)=1+p_{1} \xi+p_{2} \xi^{2}+\cdots$ analytic in the unit disc and $p(\xi) \in \mathbf{P}(C, D)$ if and only if

$$
p(\xi)=\frac{1+C w(\xi)}{1+D w(\xi)}
$$

where $w(\xi)$ is the Schwarz function. Geometrically, $p(\xi) \in \mathbf{P}(C, D)$ if and only if $p(0)=1$ and $p(\mathbf{U})$ lies inside an open disc centred with center $1-C D /\left(1-D^{2}\right)$ on the real axis having radius $C-D /\left(1-D^{2}\right)$ with diameter end points $p_{1}(-1)=1-C /(1-D)$ and $p_{1}(1)=1+C /(1+D)$. On observing that $w(\xi)=p(\xi)-1 /(p(\xi)+1)$ for $p(\xi) \in \mathbf{P}$, we have $S(\xi) \in \mathbf{P}(C, D)$ if and only if for some $p(\xi) \in \mathbf{P}$

$$
S(\xi)=\frac{(1+C) p(\xi)+1-C}{(1+D) p(\xi)+1-D} .
$$

For detailed study on the class of Janowski functions, we refer [16]. The class of Janowski star-like functions and Janowski convex functions is defined as follows:

$$
\begin{aligned}
\mathcal{S}^{*}(C, D) & :=\left\{f \in \mathscr{A}: \frac{\xi f^{\prime}(\xi)}{f(\xi)} \prec \frac{1+C \xi}{1+D \xi},-1 \leq D<C \leq 1\right\} \\
\mathscr{C}(C, D) & :=\left\{f \in \mathscr{A}:\left(1+\frac{\xi f^{\prime \prime}(\xi)}{f^{\prime}(\xi)}\right) \prec \frac{1+C \xi}{1+D \xi},-1 \leq D<C \leq 1\right\} .
\end{aligned}
$$

Inspired by the theory familiarized by Sakaguchi [17], and the study on analytic functions with respect to $(j, k)$-symmetrical points by various authors (see [18-22]), under this article, we formulate new subclasses listed in Definition 2.

Definition 2. For $-(\pi / 2)<\theta<(\pi / 2), \quad b \in \mathbb{C} \backslash\{0\}$, and $\mathscr{H}_{j, k}(\xi) / \xi \neq 0$ be defined as in (7). We say that $f \in \mathscr{K}_{s}^{b}(\vartheta ; \theta ; \psi ; g ; C, D)$ if $\mathscr{H}(\xi)=(f * g)(\xi)$ satisfies the subordination condition:

$$
\begin{aligned}
1 & +\frac{(1+i \tan \theta)}{b}\left[\frac{\vartheta \xi^{2} \mathscr{H} \prime \prime(\xi)+\xi \mathscr{H}_{\prime}(\xi)}{(1-\vartheta) \mathscr{H}_{j, k}(\xi)+\vartheta \xi \mathscr{H}_{j, k}^{\prime}(\xi)}-1\right] \\
& \prec \frac{(C+1) \psi(\xi)-(C-1)}{(D+1) \psi(\xi)-(D-1)},
\end{aligned}
$$

where $\psi \in \mathbf{P}$ and is given by

$$
\psi(\xi)=1+L_{1} \xi+L_{2} \xi^{2}+L_{3} \xi^{3}+\cdots, \quad \xi \in \mathbf{U}, L_{1} \neq 0 .
$$

Remark 1 . Here, we list few exceptional cases of the defined class $\mathscr{K}_{s}^{b}(\vartheta ; \theta ; \psi ; g ; C, D)$.
(1) If we let $C=1, D=-1, \quad \theta=0, \quad b=1$ and $g(\xi)=\xi+\sum_{n=2}^{\infty} \xi^{n}$, then $\mathscr{K}_{s}^{1}(0 ; 0 ; \psi ; g ; 1,-1) \equiv$ $\mathcal{S}_{s}^{(j, k)}(\psi)[19]$ and $\mathscr{K}_{s}^{1}(1 ; 0 ; \psi ; g ; 1,-1) \equiv \mathscr{C}_{s}^{(j, k)}(\psi)$ [19]

(2) Fixing $\quad \theta=0, \quad b=1, \quad g(\xi)=\xi+\sum_{n=2}^{\infty} \xi^{n} \quad$ and $\psi(\xi)=1+\xi /(1-\xi)$, then $\mathscr{K}_{s}^{b}(\vartheta ; 0 ; \psi ; g ; C, D)$ reduces to the class $\mathcal{S}^{(j, k)}(C, D)$ ([18], Definition 5)

For completeness, we will now define $q$-analogue of the as follows.

Definition 3. For $-(\pi / 2)<\theta<(\pi / 2), \quad b \in \mathbb{C} \backslash\{0\} \quad$ and $\mathscr{H}_{j, k}(\xi) / \xi \neq 0$ be defined as in (7). We say that $f \in \mathcal{Q} \mathscr{K}_{s}^{b}(\vartheta ; \theta ; \psi ; g ; C, D)$ if $\mathscr{H}(\xi)=(f * g)(\xi)$ holds the subordination condition:

$$
\begin{aligned}
1 & +\frac{(1+i \tan \theta)}{b}\left[\frac{\vartheta q \xi^{2} \mathfrak{D}_{q}^{2}(\mathscr{H}(\xi))+\xi \mathfrak{D}_{q} \mathscr{H}(\xi)}{(1-\vartheta) \mathscr{H}_{j, k}(\xi)+\vartheta \xi \mathfrak{D}_{q} \mathscr{H}_{j, k}(\xi)}-1\right] \\
& \prec \frac{(C+1) \psi(\xi)-(C-1)}{(D+1) \psi(\xi)-(D-1)},
\end{aligned}
$$

where $\psi \in \mathbf{P}$ and $\psi$ is defined as in (21).

By letting $\quad \psi(\xi)=1+\xi /(1-q \xi), q \in(0,1) \quad$ in $Q \mathscr{K}_{s}^{b}(\vartheta ; \theta ; \psi ; g ; C, D)$, we have

$$
\begin{aligned}
1+ & \frac{(1+i \tan \theta)}{b}\left[\frac{\vartheta q \xi^{2} \mathfrak{D}_{q}^{2}(\mathscr{H}(\xi))+\xi \mathfrak{D}_{q} \mathscr{H}(\xi)}{(1-\vartheta) \mathscr{H}_{j, k}(\xi)+\vartheta \xi \mathfrak{D}_{q} \mathscr{H}_{j, k}(\xi)}-1\right] \\
& =\frac{(C+1) w(\xi)+2+(C-1) q w(\xi)}{(D+1) w(\xi)+2+(D-1) q w(\xi)}
\end{aligned}
$$

where $q \in(0,1), w(\xi)$ is analytic in $\mathbf{U}$, and $w(0)=0$, $|w(\xi)|<1$.

Remark 2. The impact of Janowski functions on a particular conic region was initiated by Noor and Malik [23] and was subsequently studied by various authors (see [11, 24, 25] and references provided therein).

\section{Inclusion Relationships and Integral Representations of the Classes} $\mathscr{K}_{s}^{b}(\boldsymbol{9} ; \boldsymbol{\theta} ; \boldsymbol{\psi} ; \boldsymbol{g} ; C, D)$ and $\mathcal{Q}^{b}(\boldsymbol{\vartheta} ; \boldsymbol{\theta} ; \boldsymbol{\psi} ; \boldsymbol{g} ; C, D)$

Let us begin with the following.

Theorem 1. Let $F_{j, k}(\vartheta ; \xi)=(1-\vartheta) \mathscr{H}_{j, k}(\xi)+\vartheta \xi \mathscr{H}_{j, k}^{\prime}(\xi)$. If $f \in \mathscr{K}_{s}^{b}(\vartheta ; \theta ; \psi ; g ; C, D)$, then

$$
\mathfrak{R}\left[1+\frac{k(1+i \tan \theta)}{b}\left(\frac{\xi F_{j, k}^{\prime}(\vartheta ; \xi)}{F_{j, k}(\vartheta ; \xi)}-\frac{1}{k}\right)\right]>0 .
$$

Proof. From the definition of $\mathscr{K}_{s}^{b}(\vartheta ; \theta ; \psi ; h ; C, D)$ and (18), we have 


$$
\Re\left[1+\frac{(1+i \tan \theta)}{b}\left(\frac{\vartheta \xi^{2} \mathscr{H}_{\prime \prime}(\xi)+\xi \mathscr{H}_{\prime}(\xi)}{(1-\vartheta) \mathscr{H}_{j, k}(\xi)+\vartheta \xi \mathscr{H}_{j, k}^{\prime}(\xi)}-1\right)\right]>0 . \quad \begin{array}{r}
\text { Replacing } \xi \text { by } \mathcal{E}^{v} \xi \text { in } \quad(25), \quad \text { then for all } \\
\nu=0,1,2, \ldots, k-1, \text { we have }
\end{array}
$$

$$
\mathfrak{R}\left[1+\frac{(1+i \tan \theta)}{b}\left(\frac{\varepsilon^{2 v} \vartheta \xi^{2} \mathscr{H} \|\left(\varepsilon^{v} \xi\right)+\varepsilon^{v} \xi \mathscr{H}^{\prime}\left(\varepsilon^{v} \xi\right)}{(1-\vartheta) \mathscr{H}_{j, k}\left(\varepsilon^{v} \xi\right)+\vartheta \varepsilon^{\nu} \xi \mathscr{H}_{j, k}^{\prime}\left(\varepsilon^{v} \xi\right)}-1\right)\right]>0, \quad(\xi \in \mathbf{U})
$$

Using (5) in (26), we get

$$
\mathfrak{R}\left[1+\frac{(1+i \tan \theta)}{b}\left(\frac{\varepsilon^{2 v} \vartheta \xi^{2} \mathscr{H} \|\left(\varepsilon^{\nu} \xi\right)+\varepsilon^{\nu} \xi \mathscr{H}^{\prime}\left(\varepsilon^{\nu} \xi\right)}{(1-\vartheta) \varepsilon^{\nu j} \mathscr{H}_{j, k}(\xi)+\vartheta \varepsilon^{\nu j} \xi \mathscr{H}_{j, k}^{\prime}(\xi)}-1\right)\right]>0, \quad(\xi \in \mathbf{U})
$$

Suppose $v=0,1,2, \ldots, k-1$ in (27), respectively, and summing them, we arrive at

$$
\Re\left[1+\frac{(1+i \tan \theta)}{b}\left(\frac{\vartheta \xi^{2} \sum_{\nu=0}^{k-1} \varepsilon^{2 v-v j} \mathscr{H} \|\left(\varepsilon^{\nu} \xi\right)+\xi \sum_{\nu=0}^{k-1} \varepsilon^{\nu-v j} \mathscr{H} \prime\left(\varepsilon^{\nu} \xi\right)}{(1-\vartheta) \mathscr{H}_{j, k}(\xi)+\vartheta \xi \mathscr{H}_{j, k}^{\prime}(\xi)}-1\right)\right]>0
$$

or equivalently,

$$
\mathfrak{R}\left[1+\frac{k(1+i \tan \theta)}{b}\left(\frac{\xi F_{j, k}^{\prime}(\vartheta ; \xi)}{F_{j, k}(\vartheta ; \xi)}-\frac{1}{k}\right)\right]>0, \quad(\xi \in \mathbf{U}) .
$$

Hence the proof.

Now, by using the following two equivalent forms (see ([14], page 3$)$ ) of product rule of the $q$-difference operator,

$$
\begin{aligned}
\mathfrak{D}_{q}[f(\xi) g(\xi)] & =g(\xi) \mathfrak{D}_{q}[f(\xi)]+f(q \xi) \mathfrak{D}_{q}[g(\xi)] \\
& =g(q \xi) \mathfrak{D}_{q}[f(\xi)]+f(\xi) \mathfrak{D}_{q}[g(\xi)],
\end{aligned}
$$

we can establish the following result by retracing the steps as in Theorem 1.

Theorem 2. Let $\quad F_{j, k}(\vartheta ; \xi)=(1-\vartheta) \mathscr{H}_{j, k}(\xi)+\vartheta \xi \mathfrak{D}_{q}$ $\left[\mathscr{H}_{j, k}(\xi)\right]$, where $H_{j, k}(\xi) / \xi \neq 0$. If $f \in \mathcal{Q}^{2} \mathscr{K}_{s}^{b}(\vartheta ; \theta ; \psi ; g$; $C, D)$, then

$$
\mathfrak{R}\left[1+\frac{k(1+i \tan \theta)}{b}\left(\frac{\xi \mathfrak{D}_{q}\left[F_{j, k}(\vartheta ; \xi)\right]}{F_{j, k}(\vartheta ; \xi)}-\frac{1}{k}\right)\right]>0 .
$$

Theorem 3. Let $f \in \mathscr{K}_{s}^{b}(\vartheta ; \theta ; \psi ; g ; C, D)$, then

$$
\begin{aligned}
& \mathscr{H}_{j, k}(\xi)=\xi \exp \left\{\frac{1}{k} \sum_{\nu=0}^{k-1} \int_{0}^{\varepsilon^{v} \xi} \frac{b(C-D)[\psi(w(t))-1]}{(1+i \tan \theta) t[(D+1) \psi(w(t))-(D-1)]} \mathrm{d} t\right\}, \quad \text { if } \vartheta=0, \\
& \mathscr{H}_{j, k}(\xi)=\int_{0}^{\xi} \exp \left\{\frac{1}{k} \sum_{\nu=0}^{k-1} \int_{0}^{\varepsilon^{\nu} \zeta} \frac{b(C-D)[\psi(w(t))-1]}{(1+i \tan \theta) t[(D+1) \psi(w(t))-(D-1)]} \mathrm{d} t\right\} \mathrm{d} \zeta, \quad \text { if } \vartheta=1,
\end{aligned}
$$

where $\mathscr{H}_{j, k}(\xi)$ is given by (7), $w(\xi)$ is analytic in $\mathbf{U}$, and $w(0)=0,|w(\xi)|<1$.

Proof. Let $f \in \mathscr{K}_{s}^{b}(\vartheta ; \theta ; \psi ; g ; C, D)$. In view of $(20)$, we have

$$
\begin{aligned}
& \frac{\vartheta \xi^{2} \mathscr{H} \|(\xi)+\xi \mathscr{H}_{\prime}(\xi)}{(1-\vartheta) \mathscr{H}_{j, k}(\xi)+\vartheta \xi \mathscr{H}_{j, k}^{\prime}(\xi)}-1 \\
& =\frac{b(C-D)[\psi(w(\xi))-1]}{(1+i \tan \theta)[(D+1) \psi(w(\xi))-(D-1)]},
\end{aligned}
$$


where $w(\xi)$ is analytic in $\mathbf{U}$ and $w(0)=0,|w(\xi)|<1$. Substituting $\xi$ by $\varepsilon^{v} \xi$ in equality (33) and ensuing the steps as in Theorem 1, we get

$$
\frac{\xi F_{j, k}^{\prime}(\vartheta ; \xi)}{F_{j, k}(\vartheta ; \xi)}-1=\frac{1}{k} \sum_{\nu=0}^{k-1} \frac{b(C-D)\left[\psi\left(w\left(\varepsilon^{\nu} \xi\right)\right)-1\right]}{(1+i \tan \theta)\left[(D+1) \psi\left(w\left(\varepsilon^{\nu} \xi\right)\right)-(D-1)\right]} .
$$

$$
\frac{\xi F_{j, k}^{\prime}(\vartheta ; \xi)}{F_{j, k}(\vartheta ; \xi)}-\frac{1}{\xi}=\frac{1}{k} \sum_{\nu=0}^{k-1} \frac{b(C-D)\left[\psi\left(w\left(\varepsilon^{\nu} \xi\right)\right)-1\right]}{(1+i \tan \theta) \xi\left[(D+1) \psi\left(w\left(\varepsilon^{\nu} \xi\right)\right)-(D-1)\right]}
$$

Upon integration, we get

$$
\log \left\{\frac{F_{j, k}(\vartheta ; \xi)}{\xi}\right\}=\frac{1}{k} \sum_{\nu=0}^{k-1} \int_{0}^{\xi} \frac{b(C-D)\left[\psi\left(w\left(\varepsilon^{\nu} \zeta\right)\right)-1\right]}{(1+i \tan \theta) \zeta\left[(D+1) \psi\left(w\left(\varepsilon^{\nu} \zeta\right)\right)-(D-1)\right]} \mathrm{d} \zeta .
$$

or equivalently,

$$
(1-9) \mathscr{H}_{j, k}(\xi)+9 \xi \mathscr{H}_{j, k}^{\prime}(\xi)=\xi \exp \left\{\frac{1}{k} \sum_{\gamma=0}^{k-1} \int_{0}^{\varepsilon^{\prime} \xi} \frac{b(C-D)[\psi(w(t))-1]}{(1+i \tan \theta) t[(D+1) \psi(w(t))-(D-1)]} \mathrm{d} t\right\} .
$$

This concludes the proof of Theorem 3 .

Theorem 4. Let $f \in \mathscr{Q}_{s}^{b}(\vartheta ; \theta ; \psi ; g ; C, D)$, then we have

$$
\begin{aligned}
& \mathscr{H}_{j, k}(\xi)=\xi \exp \left\{\frac{\ln q}{(q-1) k} \sum_{\nu=0}^{k-1} \int_{0}^{\varepsilon^{\nu} \xi} \frac{b(C-D)[\psi(w(t))-1]}{(1+i \tan \theta) t[(D+1) \psi(w(t))-(D-1)]} \mathrm{d}_{q} t\right\}, \quad(\text { if } \vartheta=0), \\
& \mathscr{H}_{j, k}(\xi)=\int_{0}^{\xi} \exp \left\{\frac{\ln q}{(q-1) k} \times \sum_{\nu=0}^{k-1} \int_{0}^{\varepsilon^{\nu} \zeta} \frac{b(C-D)[\psi(w(t))-1]}{(1+i \tan \theta) t[(D+1) \psi(w(t))-(D-1)]} \mathrm{d}_{q} t\right\} \mathrm{d}_{q} \zeta, \quad(\text { if } \vartheta=1) .
\end{aligned}
$$

where $\mathscr{H}_{j, k}(\xi)$ defined by equality $(7), w(\xi)$ is analytic in $\mathbf{U}$, and $w(0)=0,|w(\xi)|<1$.
Proof. Let $f \in Q \mathscr{K}_{s}^{b}(\vartheta ; \theta ; \psi ; g ; C, D)$. In sight of Theorems 2 and 3 , we have

$$
\frac{\xi F_{j, k}^{\prime}(\vartheta ; \xi)}{F_{j, k}(\vartheta ; \xi)}-\frac{1}{\xi}=\frac{1}{k} \sum_{\nu=0}^{k-1} \frac{b(C-D)\left[\psi\left(w\left(\varepsilon^{\nu} \xi\right)\right)-1\right]}{(1+i \tan \theta) \xi\left[(D+1) \psi\left(w\left(\varepsilon^{\nu} \xi\right)\right)-(D-1)\right]}
$$

where $w(\xi)$ is analytic in $\mathbf{U}$ and $w(0)=0,|w(\xi)|<1$. For $f \in \mathscr{H}(\mathbf{U})$ and $0<q<1$, we obtain (see [10])

$$
I_{q} \frac{\mathfrak{D}_{q} f(\xi)}{f(\xi)}=\frac{q-1}{\ln q} \log f(\xi)
$$


where $I_{q} f$ is the Jackson $q$-integral, defined as in (14). Integrating the above equality, we get

$$
\frac{q-1}{\ln q} \log \left\{\frac{F_{j, k}(\vartheta ; \xi)}{\xi}\right\}=\frac{1}{k} \sum_{\nu=0}^{k-1} \int_{0}^{\xi} \frac{b(C-D)\left[\psi\left(w\left(\varepsilon^{\nu} \zeta\right)\right)-1\right]}{(1+i \tan \theta) \zeta\left[(D+1) \psi\left(w\left(\varepsilon^{\nu} \zeta\right)\right)-(D-1)\right]} \mathrm{d}_{q} \zeta
$$

or equivalently,

$$
(1-9) \mathscr{H}_{j, k}(\xi)+\vartheta \xi \mathscr{H}_{j, k}^{\prime}(\xi)=\xi \exp \left\{\frac{\ln q}{(q-1) k} \sum_{\nu=0}^{k-1} \int_{0}^{\varepsilon^{\nu} \xi} \frac{b(C-D)[\psi(w(t))-1]}{(1+i \tan \theta) t[(D+1) \psi(w(t))-(D-1)]} \mathrm{d}_{q} t\right\}
$$

This concludes the proof of Theorem 3 .

By fixing $C=1, D=-1, \quad \theta=0, \quad b=1, \quad$ and $g(\xi)=\xi+\sum_{n=2}^{\infty} \xi^{n}$ in Theorem 3, we state the subsequent result.

Corollary 1 (see ([19], Theorems 3 and 4$)$ ). Let $f_{j, k}(\xi) \neq 0$ be assumed as in (4).

(i) If $f \in \mathcal{S}_{s}^{(j, k)}(\phi)$, then

$$
f_{j, k}(\xi)=\xi \exp \left\{\frac{1}{k} \sum_{\nu=0}^{k-1} \int_{0}^{\varepsilon^{\nu} \xi} \frac{\phi(w(t))-1}{t} \mathrm{~d} t\right\} .
$$

(ii) If $f \in \mathscr{C}_{s}^{(j, k)}(\phi)$, then

$$
f_{j, k}(\xi)=\int_{0}^{\xi} \exp \left\{\frac{1}{k} \sum_{\nu=0}^{k-1} \int_{0}^{\varepsilon^{\nu} \xi} \frac{\phi(w(t))-1}{t} \mathrm{~d} t\right\} \mathrm{d} \text { xieta },
$$

where $w(\xi)$ is analytic in $\mathbf{U}$ and $w(0)=0,|w(\xi)|<1$.

Corollary 2. Let $f_{j, k}(\xi) \neq 0$ be assumed as in (4). If $f \in \mathcal{S}^{(j, k)}(C, D)$, then

$$
f_{j, k}(\xi)=\xi \exp \left\{\frac{1}{k} \sum_{\nu=0}^{k-1} \int_{0}^{\varepsilon^{\nu} \xi} \frac{1}{t}\left[\frac{1+C w(t)}{1+D w(t)}\right] \mathrm{d} t\right\},
$$

where $w(\xi)$ is analytic in $\mathbf{U}$ and $w(0)=0,|w(\xi)|<1$.

\section{Coefficient Inequalities for}

$$
\mathscr{K}_{s}^{b}(\boldsymbol{9} ; \boldsymbol{\theta} ; \boldsymbol{\psi} ; \boldsymbol{g} ; C, D) \text { and } \mathbb{Q} \mathscr{K}_{s}^{b}(\boldsymbol{9} ; \boldsymbol{\theta} ; \boldsymbol{\psi} ; \boldsymbol{g} ; C, D)
$$

The coefficient estimate $\left|a_{n}\right|$ of the defined function classes is determined in this section.

Theorem 5. Let $|\psi(\xi)|<|D-1 /(D+1)|, \forall \xi \in \mathbf{U}$ and $\Upsilon_{n}$ 's be real. If $f \in \mathscr{K}_{s}^{b}(\vartheta ; \theta ; \psi ; g ; C, D)$, then for $n \geq 2$,

$$
\left|a_{n}\right| \leq \frac{1}{\Upsilon_{n}} \prod_{m=1}^{n-1} \frac{\left|[(1-\vartheta)+\vartheta m] \Lambda_{m, j}(C-D) L_{1} b-2(1+i \tan \theta)\left[\vartheta m\left(m-1-\Lambda_{m, j}\right)+m-(1-\vartheta) \Lambda_{m, j}\right] D\right|}{2 \sec \theta\left[\vartheta(m+1)\left(m-\Lambda_{m+1, j}\right)+(m+1)-(1-\vartheta) \Lambda_{m+1, j}\right]} .
$$

Proof. By the definition of $\mathscr{K}_{s}^{b}(\vartheta ; \theta ; \psi ; g ; C, D)$, we have

$$
1+\frac{(1+i \tan \theta)}{b}\left[\frac{\vartheta \xi^{2} \mathscr{H}_{\prime \prime}(\xi)+\xi \mathscr{H}_{\prime}(\xi)}{(1-\vartheta) \mathscr{H}_{j, k}(\xi)+\vartheta \xi \mathscr{H}_{j, k}^{\prime}(\xi)}-1\right]=p(\xi),
$$

where $p(\xi) \in \mathbf{P}$ and satisfies the condition $p(\xi) \prec((C+1) \psi$ $(\xi)-(C-1)) /((D+1) \psi(\xi)-(D-1))$.

Equivalently, (47) can be rewritten as

$$
\begin{aligned}
& (1+i \tan \theta)\left[\sum_{n=2}^{\infty}\left[\vartheta n\left(n-1-\Lambda_{n, j}\right)+n-(1-\vartheta) \Lambda_{n, j}\right] \Upsilon_{n} a_{n} \xi^{n}\right] \\
& =b\left[\sum_{n=1}^{\infty}[(1-\vartheta)+\vartheta n] \Lambda_{n, j} a_{n} \Upsilon_{n} \xi^{n}\right]\left[\sum_{n=1}^{\infty} p_{n} \xi^{n}\right], \quad\left(a_{1}=\Upsilon_{1}=\Lambda_{1, j}=1\right) .
\end{aligned}
$$

On equating the coefficient of $\xi^{n}$, we get

$$
\begin{aligned}
& (1+i \tan \theta)\left[\vartheta n\left(n-1-\Lambda_{n, j}\right)+n-(1-\vartheta) \Lambda_{n, j}\right] \Upsilon_{n} a_{n} \\
= & b\left[p_{n-1} \Lambda_{1, j}+p_{n-2}(1+\vartheta) \Lambda_{2, j} \Upsilon_{2} a_{2}+\cdots+p_{1}\right. \\
& \left.\cdot(1+(n-2) \vartheta) \Upsilon_{n-1} a_{n-1}\right] .
\end{aligned}
$$

From Lemma 6 of [26], we have $\left|p_{n}\right| \leq\left|L_{1}\right|(C-D) / 2$, $n \geq 1$. On computation, we have

$$
\begin{aligned}
\left|a_{n}\right| \leq & \frac{|b|(C-D)\left|L_{1}\right|}{2 \sec \theta\left[\vartheta n\left(n-1-\Lambda_{n, j}\right)+n-(1-\vartheta) \Lambda_{n, j}\right] \Upsilon_{n}} \\
& \cdot\left[\sum_{m=1}^{n-1}[(1-\vartheta)+\vartheta m] \Lambda_{m, j} \Upsilon_{m}\left|a_{m}\right|\right] .
\end{aligned}
$$


Taking $n=2$, in (50), we get

$$
\left|a_{2}\right| \leq \frac{|b|(C-D)\left|L_{1}\right|}{2 \sec \theta\left[2 \vartheta\left(1-\Lambda_{2, j}\right)+2-(1-\vartheta) \Lambda_{2, j}\right] \Upsilon_{2}} .
$$

On substituting $n=2$ in (46), we can see the hypothesis is true for $n=2$. Now, taking $n=3$ in (50), we get

$$
\begin{aligned}
\left|a_{3}\right| & \leq \frac{|b|(C-D)\left|L_{1}\right|}{2 \sec \theta\left[3 \vartheta\left(2-\Lambda_{3, j}\right)+3-(1-\vartheta) \Lambda_{3, j}\right] \Upsilon_{3}}\left[1+(1+\vartheta) \Lambda_{2, j} \Upsilon_{2}\left|a_{2}\right|\right] \\
& \leq \frac{|b|(C-D)\left|L_{1}\right|}{2 \sec \theta\left[3 \vartheta\left(2-\Lambda_{3, j}\right)+3-(1-\vartheta) \Lambda_{3, j}\right] \Upsilon_{3}}\left[1+\frac{(1+\vartheta) \Lambda_{2, j}|b|(C-D)\left|L_{1}\right|}{2 \sec \theta\left[2 \vartheta\left(1-\Lambda_{2, j}\right)+2-(1-\vartheta) \Lambda_{2, j}\right]}\right] .
\end{aligned}
$$

If we let $n=3$, in (46), we have

$$
\begin{aligned}
\left|a_{3}\right| & \leq \frac{1}{\Upsilon_{3}}\left[\frac{|b|(C-D)\left|L_{1}\right| \Lambda_{1, j}}{2 \sec \theta\left[2 \vartheta\left(1-\Lambda_{2, j}\right)+2-(1-\vartheta) \Lambda_{2, j}\right]} \times \frac{\left|(C-D) L_{1} b(1+\vartheta) \Lambda_{2, j}-2(1+i \tan \theta)\left[2 \vartheta\left(1-\Lambda_{2, j}\right)+2-(1-\vartheta) \Lambda_{2, j}\right] D\right|}{2 \sec \theta\left[3 \vartheta\left(2-\Lambda_{3, j}\right)+3-(1-\vartheta) \Lambda_{3, j}\right]}\right] \\
& \leq \frac{1}{\Upsilon_{3}}\left[\frac{|b|(C-D)\left|L_{1}\right| \Lambda_{1, j}}{2 \sec \theta\left[3 \vartheta\left(2-\Lambda_{3, j}\right)+3-(1-\vartheta) \Lambda_{3, j}\right]} \times \frac{|b|(C-D)\left|L_{1}\right|(1+\vartheta) \Lambda_{2, j}+2 \sec \theta\left[2 \vartheta\left(1-\Lambda_{2, j}\right)+2-(1-\vartheta) \Lambda_{2, j}\right]|D|}{2 \sec \theta\left[2 \vartheta\left(1-\Lambda_{2, j}\right)+2-(1-\vartheta) \Lambda_{2, j}\right]}\right] \\
& \leq \frac{|b|(C-D)\left|L_{1}\right|}{2 \sec \theta\left[3 \vartheta\left(2-\Lambda_{3, j}\right)+3-(1-\vartheta) \Lambda_{3, j}\right] \Upsilon_{3}}\left[1+\frac{(1+\vartheta) \Lambda_{2, j}|b|(C-D)\left|L_{1}\right|}{2 \sec \theta\left[2 \vartheta\left(1-\Lambda_{2, j}\right)+2-(1-\vartheta) \Lambda_{2, j}\right]}\right] .
\end{aligned}
$$

Hence, the hypothesis of the theorem is true for $n=3$.

Now, let us suppose (46) is valid for $n=2,3, \ldots r$. On using

triangle inequality in (46), we get

$$
\left|a_{r}\right| \leq \frac{1}{\Upsilon_{r}} \prod_{m=1}^{r-1} \frac{[(1-\vartheta)+\vartheta m] \Lambda_{m, j}|b|(C-D)\left|L_{1}\right|+2 \sec \theta\left[\vartheta m\left(m-1-\Lambda_{m, j}\right)+m-(1-\vartheta) \Lambda_{m, j}\right]}{2 \sec \theta\left[\vartheta(m+1)\left(m-\Lambda_{m+1, j}\right)+(m+1)-(1-\vartheta) \Lambda_{m+1, j}\right]} .
$$

By induction hypothesis, we have

$$
\begin{aligned}
& \frac{|b|(C-D)\left|L_{1}\right|}{2 \sec \theta\left[\vartheta r\left(r-1-\Lambda_{r, j}\right)+r-(1-\vartheta) \Lambda_{r, j}\right] \Upsilon_{r}}\left[\sum_{m=1}^{r-1}[(1-\vartheta)+\vartheta m] \Lambda_{m, j} \Upsilon_{m}\left|a_{m}\right|\right] \\
& \leq \frac{1}{\Upsilon_{r}} \prod_{m=1}^{r-1} \frac{[(1-\vartheta)+\vartheta m] \Lambda_{m, j}|b|(C-D)\left|L_{1}\right|+2 \sec \theta\left[\vartheta m\left(m-1-\Lambda_{m, j}\right)+m-(1-\vartheta) \Lambda_{m, j}\right]}{2 \sec \theta\left[\vartheta(m+1)\left(m-\Lambda_{m+1, j}\right)+(m+1)-(1-\vartheta) \Lambda_{m+1, j}\right]} .
\end{aligned}
$$

From the above inequality, we have 


$$
\begin{aligned}
& \prod_{m=1}^{r} \frac{\left[[(1-\vartheta)+\vartheta m] \Lambda_{m, j}|b|(C-D)\left|L_{1}\right|+2 \sec \theta\left[\vartheta m\left(m-1-\Lambda_{m, j}\right)+m-(1-\vartheta) \Lambda_{m, j}\right]\right]}{2 \Upsilon_{r+1} \sec \theta\left[\vartheta(m+1)\left(m-\Lambda_{m+1, j}\right)+(m+1)-(1-\vartheta) \Lambda_{m+1, j}\right]} \\
& \quad \geq \frac{|b|(C-D)\left|L_{1}\right|}{2 \sec \theta\left[\vartheta(r+1)\left(r-\Lambda_{r+1, j}\right)+(r+1)-(1-\vartheta) \Lambda_{r+1, j}\right] \Upsilon_{r+1}}\left[\sum_{m=1}^{r}[(1-\vartheta)+\vartheta m] \Lambda_{m, j} \Upsilon_{m}\left|a_{m}\right|\right]
\end{aligned}
$$

which implies that inequality (46) is true for $n=r+1$. Hence the proof of the theorem.
Theorem 6. Let $|\psi(\xi)|<|D-1 /(D+1)|$ for all $\xi \in \mathbf{U}$ and $\Upsilon_{n}$ be real. If $f \in Q \mathscr{K}_{s}^{b}(\vartheta ; \theta ; \psi ; g ; C, D)$, then for $n \geq 2$,

$$
\left|a_{n}\right| \leq \frac{1}{\Upsilon_{n}} \prod_{m=1}^{n-1} \frac{\left|\left[(1-\vartheta)+\vartheta[m]_{q}\right] \Lambda_{m, j}(C-D) L_{1} b-2(1+i \tan \theta)\left[q 9[m]_{q}\left([m-1]_{q}-\Lambda_{m, j}\right)+[m]_{q}-(1-\vartheta) \Lambda_{m, j}\right] D\right|}{2 \sec \theta\left[\vartheta q[m+1]_{q}\left([m]_{q}-\Lambda_{m+1, j}\right)+[m+1]_{q}-(1-\vartheta) \Lambda_{m+1, j}\right]} .
$$

Proof. From the definition of $\mathcal{Q}_{K^{b}}^{b}(\vartheta ; \theta ; \psi ; g ; C, D)$, we have

$$
(1+i \tan \theta)\left[\sum_{n=2}^{\infty}\left[\vartheta[n]_{q}\left(q[n-1]_{q}-\Lambda_{n, j}\right)+[n]_{q}-(1-\vartheta) \Lambda_{n, j}\right] \Upsilon_{n} a_{n} \xi^{n}\right]=b\left[\sum_{n=1}^{\infty}\left\{(1-\vartheta)+\vartheta[n]_{q}\right\} \Lambda_{n, j} a_{n} \Upsilon_{n} \xi^{n}\right]\left[\sum_{n=1}^{\infty} p_{n} \xi^{n}\right]
$$

Equating the coefficient of $\xi^{n}$ and retracing the steps as in Theorem 5, we get

$$
\begin{aligned}
\left|a_{n}\right| \leq & \frac{|b|(C-D)\left|L_{1}\right|}{2 \sec \theta\left[\vartheta[n]_{q}\left(q[n-1]_{q}-\Lambda_{n, j}\right)+[n]_{q}-(1-\vartheta) \Lambda_{n, j}\right] \Upsilon_{n}} \\
& \times\left[\sum_{m=1}^{n-1}\left[(1-\vartheta)+\vartheta[m]_{q}\right] \Lambda_{m, j} \Upsilon_{m}\left|a_{m}\right|\right] .
\end{aligned}
$$

Now, by repeating the processes in Theorem 5, we acquire the required outcome.

If we let $\psi(\xi)=1+\xi /(1-q \xi)$ in Theorem 6 , we have the following.

Corollary 3. Let $f \in \mathscr{Q}_{K_{s}^{b}}^{b}(\vartheta ; \theta ; \psi ; g ; C, D)$ and $\Upsilon_{n}$ be real, then for $n \geq 2$,

$\left|a_{n}\right| \leq \frac{1}{\Upsilon_{n}} \prod_{m=1}^{n-1} \frac{\left|\left[(1-\vartheta)+\vartheta[m]_{q}\right] \Lambda_{m, j}(C-D)(1+q) b-2(1+i \tan \theta)\left[q \vartheta[m]_{q}\left([m-1]_{q}-\Lambda_{m, j}\right)+[m]_{q}-(1-\vartheta) \Lambda_{m, j}\right][D(1+q)+(1-q)]\right|}{2 \sec \theta\left[\vartheta q[m+1]_{q}\left([m]_{q}-\Lambda_{m+1, j}\right)+[m+1]_{q}-(1-\vartheta) \Lambda_{m+1, j}\right]}$.

If we let $\theta=0, b=1, g(\xi)=\xi+\sum_{n=2}^{\infty} \xi^{n}$, and $\psi(\xi)=$ $1+\xi / 1-\xi$ in Theorem 5 , we have the following.

Corollary 4. (see [18], Theorem 2) Let $f \in \mathcal{S}^{(j, k)}(C, D)$ and $\Upsilon_{n}$ be real, then for $n \geq 2$,

$$
\left|a_{n}\right| \leq \prod_{m=1}^{n-1} \frac{\Lambda_{m, j}[(C-D)-1]+m}{(m+1)-\Lambda_{m+1, j}} .
$$

\section{Conclusion}

Very few studies have been showed on analytic functions with regard to $(j, k)$-symmetric points. Since we have articulated the problem differently so as to deviate from the similar studies, only few special cases could be discussed. Furthermore, by swapping the ordinary differentiation with quantum differentiation, we have tried at the discretization of some of the familiar findings. 


\section{Data Availability}

No data were used to support this study.

\section{Conflicts of Interest}

The authors declare that there are no conflicts of interest.

\section{References}

[1] A. W. Goodman, Univalent Functions, Mariner, Tampa, FL, USA, 1983.

[2] P. Liczberski and J. Połubiński, "On $(j, k)$-symmetrical functions," Mathematica Bohemica, vol. 120, no. 1, pp. 13-28, 1995.

[3] J. Dziok and H. M. Srivastava, "Classes of analytic functions associated with the generalized hypergeometric function," Applied Mathematics and Computation, vol. 103, no. 1, pp. 1-13, 1999.

[4] G. Ş. Sălăgean, "Subclasses of univalent functions, in complex analysis-fifth Romanian-Finnish seminar," Lecture Notes in Mathematics, Springer, Berlin, Germany, 1981.

[5] T. M. Seoudy, "Certain subclasses of spiral-like functions associated with $q$-analogue of Carlson-Shaffer operator," AIMS Mathematics, vol. 6, no. 3, pp. 2525-2538, 2021

[6] F. H. Jackson, "On q-definite integrals," Journal of Pure and Applied Mathematics Quarterly, vol. 41, pp. 193-203, 1910.

[7] F. H. Jackson, "XI.-on q-Functions and a certain difference operator," Transactions of the Royal Society of Edinburgh, vol. 46, no. 2, pp. 253-281, 1909.

[8] H. M. Srivastava, K. Bilal, H. K. Nazar, and A. Qazi Zahoor, "Coefficient inequalities for $q$-starlike functions associated with the Janowski functions," Hokkaido Mathematical Journal, vol. 48, no. 2, pp. 407-425, 2019.

[9] S. Kanas and D. Răducanu, "Some class of analytic functions related to conic domains," Mathematica Slovaca, vol. 64, no. 5, pp. 1183-1196, 2014

[10] S. Agrawal and S. K. Sahoo, "A generalization of starlike functions of order alpha," Hokkaido Mathematical Journal, vol. 46, no. 1, pp. 15-27, 2017.

[11] M. Arif, O. Barukab, H. M. Srivastava, S. Abdullah, and S. A. Khan, "Some Janowski type harmonic q-starlike functions associated with symmetrical points," Mathematics, vol. 8 , no. 4 , p. $629,2020$.

[12] M. H. Annaby and Z. S. Mansour, " $q$-fractional calculus and equations," Lecture Notes in Mathematics, Springer, Berlin, Germany, 2012.

[13] A. Aral, V. Gupta, and R. P. Agarwal, Applications of QCalculus in Operator Theory, Springer, Berlin, Germany, 2013.

[14] V. Kac and P. Cheung, Quantum Calculus, Universitext, Springer-Verlag, Berlin, Germany, 2002.

[15] W. C. Ma and D. Minda, "A unified treatment of some special classes of univalent functions," in Proceedings of the Conference on Complex Analysis, Oberwolfach, Germany, August 1992.

[16] W. Janowski, "Some extremal problems for certain families of analytic functions I," Annales Polonici Mathematici, vol. 28, no. 3, pp. 297-326, 1973.

[17] K. Sakaguchi, “On a certain univalent mapping," Journal of the Mathematical Society of Japan, vol. 11, pp. 72-75, 1959.

[18] F. S. M. Al Sarari, B. A. Frasin, T. Al-Hawary, and S. Latha, "A few results on generalized Janowski type functions associated with $(j, k)$-symmetrical functions," Acta Universitatis Sapientiae, Mathematica, vol. 8, no. 2, pp. 195-205, 2016.
[19] K. R. Karthikeyan, "Some classes of analytic functions with respect to $(j, k)$-symmetric points," Romai Journal, vol. 9, no. 1, pp. 51-60, 2013.

[20] K. R. Karthikeyan, K. Srinivasan, and K. Ramachandran, "On a class of multivalent starlike functions with a bounded positive real part," Palestine Journal of Mathematics, vol. 5, no. 1, pp. 59-64, 2015.

[21] C. Selvaraj, K. R. Karthikeyan, and G. Thirupathi, "Multivalent functions with respect to symmetric conjugate points," Punjab University Journal of Mathematics, vol. 46, no. 1, pp. 1-8, 2014.

[22] H. M. Srivastava, S. Z. H. Bukhari, and M. Nazir, "A subclass of $\alpha$-convex functions with respect to $(2 j, k)$-symmetric conjugate points," Bulletin of the Iranian Mathematical Society, vol. 44, no. 5, pp. 1227-1242, 2018.

[23] K. I. Noor and S. N. Malik, "On coefficient inequalities of functions associated with conic domains," Computers \& Mathematics with Applications, vol. 62, no. 5, pp. 2209-2217, 2011.

[24] F. M. Al-Oboudi, "On univalent functions defined by a generalized Sălăgean operator," International Journal of Mathematics and Mathematical Sciences, vol. 2004, no. 25-28, 1436 pages, Article ID 172525, 2004.

[25] H. M. Srivastava, "Univalent functions, fractional calculus and associated generalized hypergeometric functions," in Univalent Functions, Fractional Calculus and Their Applications, H. M. Srivastava and S. Owa, Eds., pp. 329-354, John Wiley and Sons, Hoboken, NJ, USA, 1989.

[26] K. R. Karthikeyan, G. Murugusundaramoorthy, and T. Bulboacă, "Properties of $\lambda$-pseudo-starlike functions of complex order defined by subordination," Axioms, vol. 10, no. 2 , p. 86,2021 . 